\title{
Chlorogenic Acid and Geniposide Combination Prevents NASH in Rats Fed High-fat diet via Microbiota and TLR4-LPS Pathway
}

Zhijia Zhou

Xiamen University

Lingxia Xu

Xiamen University

Shaoliang Zhang

Xiamen University

Shilin Xu

Xiamen University

Yanmiao Yang

Xiamen University

Ning Qu

Xiamen University

Penghua Lai

Xiamen University

Xiaoying Li

Xiamen University

Ting Zhang

Xiamen University

Xue Sun

Xiamen University

Liangqin Gao

Hospital of Xiamen University

Dawei Lu

Xiamen University

Yujie Wang

Xiamen University

Huiqing Liang

Xiamen Hospital of Traditional Chinese Medicine

Shaodong Chen ( $\nabla$ adong@xmu.edu.cn )

Xiamen University 


\section{Research Article}

Keywords: gut microbiota, chlorogenic acid, geniposide, nonalcoholic steatohepatitis, TLR4

Posted Date: March 1st, 2021

DOI: https://doi.org/10.21203/rs.3.rs-238577/v1

License: (c) (i) This work is licensed under a Creative Commons Attribution 4.0 International License. Read Full License 


\section{Abstract}

Objective: Chlorogenic acid and geniposide (CG) are derived from traditional Chinese medicine, Yinchenhao Recipe (QCHR), and can improve the clinical efficacy of NASH patients. This study investigated the effects of CG on NASH and expounded its Potential mechanism of action through the LPS-TLR4 pathway and microbiota.

Methods: Rats were randomized into Control (C), Model (M), Chlorogenic Acid and Geniposide (CG), Pioglitazone (PH) and Bifico (B) groups. After an 8-week high-fat diet (HFD), CG, PH and B oral treatment were initiated and carried out for a further 8 weeks. The stool samples were used in a16S rDNA V4 highly variable region measurement method in order to regulate the role of $C G$ in gut microbiota. The concentrations of triglyceride (TG), cholesterol (CHO), interleukin-1 $\beta$ (IL-1 $\beta$ ), interleukin-6 (IL-6), tumor necrosis factor-a (TNF-a) in LPS were detected by the corresponding methods.

Results: Observations were made that CG significantly improved the pathology of the liver and terminal ileum tissue. The accumulation of TG and the content of inflammatory cytokines in the liver were significantly decreased and the abundance of Proteobacteria was significantly down-regulated. The expression of TLR4, AP-1, MyD88, and phosphorylated NF-kB p65 were significantly decreased. All the findings above indicated that $C G$ was highly effective in improving the composition of gut microbiota, decreasing the production of endogenous LPS, and reducing the secretion of inflammatory cytokines through the gut-liver axis.

Conclusion: CG can regulate the abundance and diversity of the intestinal microbial community and improve liver inflammation and steatosis in NASH rats by reducing LPS-TLR4-mediated inflammation.

\section{Introduction}

Non-alcoholic fatty liver disease (NAFLD) is defined as hepatic steatosis excluding the causes of significant alcohol consumption, steatogenic medication or hereditary disorders ${ }^{1}$ - It's histologically characterized by steatosis ${ }^{2}$. About $25 \%$ of adults worldwide suffer from Nonalcoholic fatty liver disease $(\mathrm{NAFLD})^{3}$ and despite the known risk factors such as diabetes, obesity, age, gender and race, the prevalence of NAFLD is still increasing ${ }^{4}$. NAFLD will elevate the risk of all-cause mortality, liver-related deaths, malignancy, diabetes and coronary artery disease ${ }^{5}$. Histologically, NAFLD can be classified into non-alcoholic fatty liver (NAFL) and non-alcoholic steatohepatitis (NASH) ${ }^{1}$. NASH, as an aggressive form of NAFLD, can develop into liver fibrosis, cirrhosis and hepatocellular carcinoma (HCC) in $5-15 \%$ of patients ${ }^{6}$. With the development of the fibrosis stage, the mortality risk of NAFLD patients increases exponentially ${ }^{7}$. Lifestyle changes are the basis of treatment for patients with NAFLD. The treatment of $\mathrm{NASH}$ can be divided into drugs that target disease pathogenesis (such as insulin resistance and de novo lipogenesis) and drugs that target downstream processes (such as cellular stress, apoptosis, inflammation and fibrosis). None of these are approved by the US Food and Drug Administration ${ }^{6,8}$. 
Chlorogenic acid and geniposide are derived from the Yinchenhao Recipe, which can improve the clinical efficacy of NASH patients ${ }^{9}$. A profusion of studies have evidenced that chlorogenic acid and geniposide have antioxidant effects on different diseases ${ }^{10-14}$. Chlorogenic acid (CGA), one of the most common and abundant polyphenols, is present in a wide variety of beverages and foods. Chlorogenic acid has anti-inflammatory and antioxidant effects on the liver ${ }^{15,16}$. There is evidence that demonstrates how chlorogenic acid can improve obesity induced by a high-fat diet by improving intestinal dysbiosis ${ }^{17}$. Geniposide is an important active ingredient of Gardenia jasminoides Ellis fruit can increase SOD levels and cell viability, reduce ALT, AST and LDH levels and confirm the significant hepatic protection ${ }^{18}$. Geniposide can also restore any impaired function of the intestinal barrier ${ }^{19}$. Our previous research evidenced that geniposide or chlorogenic acid can decrease fatty liver steatosis and serum ALT and AST level in rats with $\mathrm{NASH}$, but the mechanism was unclear ${ }^{20-22}$.

The pathogenesis of NAFLD is highly complex, the "multiple hit" hypothesis considers that insulin resistance and hormones secreted from the adipose tissue, genetic and epigenetic factors, nutritional factors and gut microbiota are all involved in NAFLD ${ }^{23}$. The nutrients, bacterial products and metabolites from the gut will be filtered upon reaching the liver. This integral link between the gut and liver is defined as the gut-liver axis. When the intestinal permeability increased, more bacterial products such as LPS crossed the epithelial barrier. LPS can be recognized by toll-like receptors (TLRs) (especially TLR4), inducing the synthesis of a variety of inflammatory cytokines, which induce inflammation, oxidative stress, and insulin resistance 24,25 .

Considering CG's antioxidant, blood fat reduction, cholesterol-lowering and prebiotic effects, the hypothesized that CG would ameliorate NAFLD through intestinal regulating was formulated. The experiment aim is to examine the regulatory effect of $C G$ on intestinal microbiota,hepatic inflammation and steatosis in high-fat-diet-induced NASH rat model.

\section{Result}

\subsection{CG attenuated HFD-induced obesity and liver injury}

After the 16 week experiment, rats in the $\mathrm{M}$ group gained more epididymal fat weight than the control group, while the $C G$ group gained less compared with the $M$ group, but there was no significant difference between the $\mathrm{PH}$ and $\mathrm{B}$ groups (Fig $1 \mathrm{~A}, \mathrm{~B}$ ). The liver wet weight and hepatic index were significantly increased in the $M$ group, respectively, compared with the $C$ group. Post intervention at 8-weeks, the liver wet weight and hepatic index in the $\mathrm{CG}$ and $\mathrm{PH}$ group were significantly decreased compared to those in the $M$ group, however, there was no significant difference between the $B$ and $M$ groups (Fig. 1C, D). The serum levels of ALT and AST were significantly increased in the M group compared with the control group and significantly decreased after the CG intervention at 8-weeks (Fig. 1E, F). 


\subsection{CG profoundly changed the composition of the intestinal microbiota in HFD-fed rats}

To reveal the effects of HFD and the administration of CG on the microbiota structure, we sequenced the bacterial 16S rRNA at baseline and after 16 weeks, OTU levels by Shannon diversity index were significantly increased in the $\mathrm{CG}, \mathrm{PH}$ and $\mathrm{B}$ groups compared with the M group (Fig.2A). Unweighted PCoA, PCoA and NMDS analyses were conducted to provide an overview of the gut microbiota composition of the five animal groups at baseline and the end of the trial. The plotted scores showed a substantial change in gut microbiota composition in rats fed an HFD. The results indicated that the HFDinduced NASH model had a considerable impact on the gut microbiota composition. CG intervention shifted the overall structure of the HFD-disrupted gut microbiota toward that of the control rats (Fig. 2BD). Venn diagrams also showed that 377 OTUs were shared between the $C$ and CG groups, 277 OTUs were shared between the $\mathrm{C}$ and $\mathrm{M}$ groups (Fig. 2E). A hierarchical cluster analysis (HCA) was conducted, generating a heatmap that provided a comprehensive overview of which groups were clustered. The heatmap showed that the distance between the $C$ and CG groups was closer than C and M's (Fig. 2F). 16 weeks of HFD feeding induced extensive changes in the gut microbial community structure at the phylum level compared with the $C$ group. There was an increase in the abundance of Firmicutes $(95.0 \%$ vs. $82.4 \%)$ and decreases in the abundances of Actinobacteria ( $0.03 \%$ vs. $0.13 \%)$, Fusobacteria $(0.007 \%$ vs. $0.002 \%$ ) and Proteobacteria ( $0.1 \%$ vs. $0.3 \%$ ). However, CG intervention mitigated the HFD-induced decrease in Actinobacteria and Proteobacteria, and the HFD-induced increase in Firmicutes. At the genus level, Streptococcus, Staphylococcus, Corynebacterium_1 were decreased in the M group compared to the C group, all of which were reversed by CG intervention. CG intervention decreased the abundances of Lactobacillus and Romboutsia compared with the HFD group (Fig. 2G, H). The plot showed that the abundance of Firmicutes, Actinobacteria and Fusobacteria had a significant difference in five groups (Fig.2I). In our experiment, the abundance of Firmicutes was much higher in the $\mathrm{M}$ group compared to the $\mathrm{C}$ group, and reversed by $\mathrm{CG}, \mathrm{PH}$ and $\mathrm{B}$ intervention (Fig. 2J). However, the abundance of Bacteroidetes was similar among the five groups (Fig.2K). The ratio of Firmicutes/Bacteroidetes (F/B) was also compared as a featured sign of obesity among the five groups. As indicated in Figure 2L, HFD significantly increased the ratio of $F / B$, and its effect can be conversed by $C G$ and $B$.

\subsection{Terminal ileum epithelial barrier damage is diminished by administration of GC in rats fed an HFD, leading to the inhibition of LPS leakage}

The HE staining demonstrated that the HFD-induced terminal ileum injury was alleviated by CG intervention (Fig.3A). The terminal ileum tissue of the rats in the $\mathrm{C}$ group was structurally intact, and the intestinal villi were neat and had an orderly arrangement. The terminal ileum tissue of rats in the $\mathrm{M}$ group presented damage to the intestinal mucosal barrier, the intestinal epithelial cells were detached, and the intestinal mucosal mechanical barrier was impaired. After treatment with CG, the ileal mucosal structure 
fully recovered, and the propria intestinal gland was abundant with goblet cells. After treatment with $\mathrm{PH}$, the intestinal villi were orderly arranged, and there was a small amount of intestinal epithelial cells detached. After treatment with $B$, an intact terminal ileum structure, rich intestinal gland, and abundance of goblet cells can be observed. The expression levels of tight junction proteins were also examined, such as Occludin and zonula occluden-1 (ZO-1). The protein levels of Occludin and ZO-1were reduced in the M group, compared to the $C$ group, and $C G$ and $B$ treatment rescued the protein expression of Occludin (Figure 3B,D) and ZO-1(Figure 3C,E) through Immunohistochemistry. The plasma level of endotoxin was determined, which is an LPS and one of the main components of the cell wall of gram-negative bacteria.. With the increase of gram-negative intestinal bacteria in the M group, the LPS content in the plasma of M group was significantly increased compared with that in the $\mathrm{C}$ group. After treatment, the LPS content in the plasma was significantly decreased in the $\mathrm{CG}$ group, but not in the $\mathrm{PH}$ group and $\mathrm{B}$ group (Fig. $3 \mathrm{~F}$ ). From these results it can be observed that CG can prevent leaking of endotoxin from the gut into the bloodstream by upregulating the expression of TJ proteins.

\section{4. +}

Hepatic steatosis is a predominant feature of NAFLD. For this reason, examined the effect of CG on $\mathrm{NASH}$ induced by the HFD diet was examined. As shown in Fig. 4A, in contrast to the $\mathrm{C}$ group, there was extensive steatosis and ballooning of hepatocytes and partial hepatocyte necrosis with a large area of nuclear fragmentation or dissolution with inflammation in the M group by H\&E. Notably, HFD-induced steatosis and ballooning in the liver were decreased by CG treatment (Fig. 4A). The oil red $O$ staining also demonstrated that HFD-induced lipid droplets in the liver were reduced by CG treatment (Fig. 4B,C). The TG content was significantly increased in the liver tissue of the $M$ group compared with that of the $C$ group. After treatment, the TG content in the liver tissue was significantly decreased in the CG group, PH

group and B group (Fig. 4D). Similarly, HFD feeding markedly enhanced cholesterol in the liver of rats and was further reversed to normal levels by $\mathrm{CG}$ and PH treatment (Fig. 4E). 
Table 1

NAFLD activity score (NAS) system

\begin{tabular}{|lll|}
\hline item & definition & score \\
\hline Steatosis & $<5 \%$ & 0 \\
\hline & $5-33 \%$ & 1 \\
& $>33-66 \%$ & 2 \\
\hline & $>66 \%$ & 3 \\
\hline Lobular inflammation & No foci & 0 \\
\hline & $<2$ foci per $200 \times$ field & 1 \\
& $2-4$ foci per $200 \times$ field & 2 \\
\hline Ballooning & None & 3 \\
\hline & Few balloon cells $200 \times$ field & 0 \\
\hline Many cells/prominent ballooning & 2 \\
\hline
\end{tabular}

\subsection{CG inhibited the activation of nuclear factor $\mathrm{KB}$ signaling and decreased the content of inflammatory cytokines (IL-1 $\beta$, IL-6, TNF-a)}

Changes in gut microbiota control metabolic LPS-induced inflammation in high-fat diet-induced insulin resistance and inflammation in mice. LPS and TLR4 signaling control the production of proinflammatory cytokines, leading to chronic inflammation and insulin resistance in HFD-fed mice 26,27 . The expression levels of TLR4, AP-1, MyD88 and phosphorylated NF-KB p65 were increased significantly in the liver tissue of the $\mathrm{M}$ group compared with that of the $\mathrm{C}$ group. After treatment, the expression levels of TLR4, AP-1, MyD88, and phosphorylated NF-KB p65 in the liver tissue were significantly decreased in the CG, PH, and $B$ group (Fig. 5A-F). These results show that HFD-fed rats produce higher levels of pro-inflammatory cytokines in hepatic and adipose tissue, including tumor necrosis factor-alpha (TNF- $a$ ) and interleukin-1beta (IL1 $\beta$ ). The concentration of these cytokines after 14 weeks of HFD feeding was measured. IL-1 $\beta$, IL6 and TNF-a content in the liver tissue of the M group were significantly increased compared with that of the $C$ group. After treatment, the IL-1 $\beta, I L-6, T N F-a$ content in the liver tissue were significantly decreased in the CG, PH and B group (Fig. 5G-I). The content of IL-1 $\beta, I L-6$, TNF-a in plasma was also detected. Notably, a similar conclusion was drawn in regards to both plasma and liver tissue (Supplement Fig 2). 
Administration of CG improves microbiota dysbiosis, increases tight junction proteins in the gut, and decreases the production of endotoxin. CG also attenuates NASH through inhibiting activation of TLR4LPS signaling and the production of proinflammatory cytokines.

\section{Discussion}

There is an increasing amount of evidence suggesting that alterations in the gut microbiota correlate with liver disease or immunological disease ${ }^{28,29}$. As high-throughput sequencing technology has progressively matured, many studies have recorded the changes in the gut microbiota of NASH individuals. Using probiotics and prebiotics to regulate gut microbiota has become a new approach to prevent and treat NAFLD ${ }^{30-32}$. In this study, the community structure of the rat fecal microbiome induced by a high-fat diet through 16s rRNA gene sequencing was demonstrated. Observations were made that intestinal microbiota and inflammatory cytokines such as TNF-a, IL-1 $\beta$ and IL-6 had significantly interfered with the 16-week HFD in rats. However, the CG intervention at 8-weeks corrected the disturbance of intestinal microbiota to a certain extent and reduced the production of LPS in plasma, while inhibiting the TLR4LPS pathway. The imbalance of inflammation cytokines and the reversal of steatohepatitis in high-fat rats was also corrected. These results suggest that CG may have major significance in the treatment of $\mathrm{NASH}$.

The pathogenesis of NAFLD is highly complex, including insulin resistance, hormones secreted from the adipose tissue, genetic and epigenetic factors, nutritional factors and gut microbiota ${ }^{23}$. Recent research into the effects of chlorogenic acid and geniposide on the gut and liver has indicated that geniposide can administer cholesterol metabolism by regulating FXR-mediated gut-liver crosstalk of bile acids ${ }^{33}$.

Research also indicated that geniposide can ameliorate barrier dysfunction via AMPK-mediated inhibition of the MLCK pathway ${ }^{19}$. Previous studies demonstrated that geniposide has beneficial effects on liver diseases both in vivo and vitro ${ }^{34,35}$. The present study concludes that the action mechanism of the CG combination on NAFLD is multi-targeting. For this reason, it is necessary to further investigate the effects of the CG combination on regulation of microbiota in NAFLD.

CG, derived from the Yinchenhao Recipe (QCHR), is a traditional Chinese medicine formula composed of capillaris (Artemisia capillaris), gardenia (Gardenia jasminoides) and rhubarb (Rheum rhabarbarum). The main active compounds of YCHR are chlorogenic acid and geniposide. YCHR is comprised of $12 \mathrm{~g}$ capillaris (Artemisia capillaris), $9 \mathrm{~g}$ gardenia (Gardenia jasminoides), and $9 \mathrm{~g}$ rhubarb (Rheum rhabarbarum $)^{36}$ In the present study, the dose given to rats equates to approximately $4 \mathrm{~g} /$ day for humans. ${ }^{37} \mathrm{PH}$ is insulin-sensitizing agents, and $\mathrm{B}$ is probiotics. $\mathrm{PH}$ is the class thiazolidinedione with the effect of enhancing insulin sensitivity and glucose utilization in peripheral tissues ${ }^{38}$. Bifico contains the viable bacteria Enterococcus faecalis(EF), Bifidobacterium longum (BL) and Lactobacillus acidophilus (L), being approved by the State Food and Drug Administration (SFDA) as an over-the-counter (OTC) drug in October $2002^{39}$. B can ameliorate the milieu of the intestine and impede the expression of inflammatory factors, such as TNF- $a$ and IL-1 $1 \beta^{40}$. 
To determine the underlying mechanism of how CG improves inflammation and obesity in rats, the gut microbiota in each group at the 16th week was detected. In the present study, observations were made that the OTU levels were significantly increased in the CG, PH and B groups compared with the $\mathrm{M}$ group according to the Shannon diversity index. Venn diagrams depicted that there were far more OTUs in the CG group than those in the $C$ and $M$ groups, and the lowest in the $M$ group. According to PCoA, NMDS and hierarchical clustering analysis, CG reversed the overall change in the intestinal flora structure induced by HFD, and CG was allocated between the $C$ and $M$ groups.

A recent study revealed that the administration of $S$. filiforme can improve physiological parameters of the heart and liver through regulating gut microbiota in HFD-induced rats, physiological parameters of the liver being closely correlated to special bacteria ${ }^{41}$. Similarly, in the present study, at the phylum level, the intestine microbiota of $\mathrm{M}$ was characterized by an increase in the abundance of Firmicutes and a decrease in the abundance of Bacteroides, Proteobacteria and Actinobacteria, which was similar to the previously reported gut microbiota in the intestines of obese people $e^{42,43}$. Prior research has suggested that Firmicutes are beneficial for obese people to obtain energy from food, and eventually gain weight ${ }^{44}$. The claim that CG can reduce the weight gain of HFD rats can be reasonably believed. This is because CG decreases the abundance of Firmicutes. I.N. Abdallah, et al showed that the ratio of Firmicutes / Bacteroidetes is elevated in the intestines of obese people ${ }^{45}$, and it was also decreased in the CG group compared to the $\mathrm{M}$ group. At the genus level, Romboutsia were decreased in the $\mathrm{M}$ group compared to the C group, all of which was reversed by CG intervention. Romboutsia belongs to Bacteria-FirmicutesClostridiaClostridiales-Peptostreptococcaceae, which is related to body energy metabolism ${ }^{46}$. The above indicates that the direct modulating effects of CG on gut bacteria may play a pivotal role in the control of obesity. Observations were made that the abundance of Streptococcus, Staphylococcus, Corynebacterium_1 was increased in the CG group. Streptococcus thermophilus, as a probiotic cocktail, can improve liver inflammation in NASH patients ${ }^{47}$. Thus, the results above demonstrate that the administration of CG remodels the structure of the gut environment and improves the gut microbiota dysbiosis induced by HFD feeding. This finding may partially explain the beneficial role of CG in microbiota in HFD-induced NASH.

Notably, the present study demonstrated that the reduction of Proteobacteria can lower the production of endogenous LPS. The immunohistochemical results of the small intestine also showed that CG can restore the intestinal barrier by up-regulating tight junction proteins and inhibit the leakage of intestinal endotoxin into the blood. The present study further demonstrated that the LPS-mediated TLR4/NF-KB pathway is integral to the pathogenesis of $\mathrm{NASH}^{48}$. $\mathrm{CG}$ directly inhibits endotoxin-induced activation of the TLR4-LPS pathway and production of proinflammatory cytokines including IL-1 $\beta$, IL-6, and TNF-a (Fig. 5). TNF-a induces hepatocyte cell death, causes insulin resistance, which results in hepatocyte steatosis, and regulates $\mathrm{KCs}$ activation through an autocrine mechanism. IL- 6 is a potential mediator of insulin resistance ${ }^{49}$. IL-1 $\beta$ induces steatosis, hepatocyte injury, and fibrosis ${ }^{50}$. The inflammation cytokines like TNF- $a$, IL-1 $\beta$, and IL- 6 are critical to the pathogenesis of NASH. The reduction of cytokine such as TNF- $a$, IL-1 $\beta$ and IL- 6 and the level of LPS in plasma through CG, PH and B was established. 
From the above, the conclusion was drawn that CG attenuated HFD-induced obesity and liver injury in rats by regulation of gut microbiota and inhibition of the TLR4-LPS pathway.

There are several limitations to the present study. Firstly, although CG can improve major indicators of $\mathrm{NASH}$ in the livers, its effect on metabolic parameters remains unclear due to the limitation of the highfat-diet-induced NASH rat model. Body weight does not decrease significantly compared with the M group after CG treatment (data not shown). Secondly, better grouping strategies can be set, such as adding a control group administrated with natural compounds. This can better clarify the impact of a high-fat diet on the intestinal flora. Chlorogenic acid and geniposide could also be added separately in individual groups to evaluate the advantages of their combination. Finally, the correlations of gut microbiome changes with physiological parameters and LPS-TLR4 pathway in rats fed a high-fat diet require further investigation.

In general, CG can reduce the abundance of bacteria producing LPS by changing the composition of the intestinal flora. CG can also increase the expression of Tj protein and reduce the entry of LPS into the blood through the intestine. This contributes to preventing liver injury through the inhibiting activation of the LPS-TLR4 pathway, decreasing the translocation of NF-KB and AP-1 and decreasing the production of inflammatory cytokines including IL-1 $\beta, \mathrm{IL}-6$, and TNF- $a$. CG can provide a therapeutic effect on NASH by targeting intestinal microbiota then manipulating intestinal microecological imbalance. The results of the present study highlight the therapeutic potential of CG in NASH management.

\section{Material And Methods}

\subsection{Plant materials and standards}

Chlorogenic acid (IUPAC name: (1S,3R,4R,5R)3-[(E)-3-(3,4-dihydroxyphenyl)prop-2-enoyl]oxy1,4,5trihydroxycyclohexane-1-carboxylic acid, purity $>98 \%$ ) and Geniposide (IUPAC name: methyl (1S,4aS,7aS)7-(hydroxymethyl)-1-[(2S,3R,4S,5S,6R)-3,4,5trihydroxy-6-(hydroxymethyl)oxan-2-yl]oxy1,4a,5,7atetrahydrocyclopenta[c]pyran-4-carboxylate, purity $>98 \%$ ) are commercial products of Nanjing ZeLang Biological Technology Co., Ltd(ZL20170409528).pioglitazone hydrochloride was purchased from Chongqing Kerui Pharmaceutical (Group) Co., Ltd(H20080271) and bifico was purchased from Shanghai shangyaoxinyi Pharmaceutical Co., Ltd(S10950032).

\subsection{Animals and treatment}

Sprague-Dawley (SD) rats (body weight $200 \pm 20 \mathrm{~g}$ ) were purchased from the Shanghai Sippe-Bk Lab Animal Co., Ltd. All rats were housed and kept under controlled conditions $\left(23 \pm 2^{\circ} \mathrm{C}\right)$, humidity $(60 \pm 10 \%)$ and a 12-hour light-dark cycle. The rats adapted to the conditions for a week and were then randomly allocated into a high-fat-diet (HFD) model group and normal diet control group ( $\mathrm{C}$ group; $\mathrm{n}=6$ ). Rats were fed according to a high-fat diet $(77.5 \%$ common feed $+0.5 \%$ sodium cholate $+2 \%$ cholesterol $+5 \%$ soya bean $+5 \%$ sucrose $+10 \%$ lard from) for 16 weeks in order to produce a NAFLD rat model ${ }^{51}$. At the beginning of the 9th week, the rats in the HFD-fed model group were randomly divided into 4 groups $(n=6$ 
in each group): Model group (M group), Chlorogenic acid and Geniposide group (CG group), Pioglitazone hydrochloride group ( $\mathrm{PH}$ group) and Bifid triple viable capsule (Bifico) group (B group) and a single rat as the experimental unit. The groups mentioned above were fed a HFD for another 8 weeks. The rats in the $C$ group were fed with common feed for 16 weeks. From the 9 th week, rats in the $\mathrm{M}$ and $\mathrm{C}$ groups were fed with double distilled water in the dose of $10 \mathrm{ml} / \mathrm{kg}$-day absorbed intact in the stomach. Rats in the CG group were fed with chlorogenic acid and geniposide aborbed intact in the stomach. Rats in the CG group were also fed a HFD for 16 weeks, followed by a daily gavage of a mixture of a dosage of $60 \mathrm{mg} / \mathrm{kg}$ geniposide and a dose of $60 \mathrm{mg} / \mathrm{kg}$-day chlorogenic acid (weight ratio $=66.7: 1$ ) in addition to a HFD for an additional 8 weeks. Rats in the PH group were fed with pioglitazone hydrochloride in the dose of 10 $\mathrm{mg} / \mathrm{kg}$-day absorbed intact in the stomach. Rats in the B group were fed with bifico in the dose of 210 $\mathrm{mg} / \mathrm{kg}$-day absorbed intact in the stomach.

\subsection{Ethics statement}

Experiments were conducted following established animal protocols and the guidelines approved by the animal experimental ethics committee of Xiamen University (approval No. XMULAC20200055).

All methods with respect to animals were carried out in accordance with relevant ARRIVE guidelines.

\subsection{Sample collection}

At the end of the 16th week, blood was extracted from the abdominal aorta in all the rats and the body weight was weighed. Rats were intraperitoneally injected with $10 \%$ chloral hydrate for euthanasia and then liver and epididymal adipose tissue samples were weighed and collected. Fecal samples were collected immediately for gut microbiota analysis. The liver and terminal ileum samples were stored in the $10 \%$ formaldehyde for histopathological analysis. The rest of the liver samples were stored at $-80^{\circ} \mathrm{C}$ condition for western blotting and biochemical analysis.

\subsection{Determination of AST, ALT in serum}

Blood samples were collected from the abdominal aorta and stored at room temperature (RT) for $1 \mathrm{~h}$. After centrifugation at $3500 \mathrm{rpm}$ for $10 \mathrm{~min}$, serum samples were collected for determination of ALT and AST levels using commercial kits (Nanjing Jiancheng Bioengineering Institute).

\subsection{Histopathological evaluation of liver and the terminal ileum tissue}

Formalin-fixed and paraffin-embedded the liver and ileum tissue, stained with hematoxylin-eosin (HE), Immunohistochemistry of ileum sections were incubated with antibodies against zonula occludens 1 (ZO-1) (1:100; GB111402, Servicebio, Wuhan, China) and Occludin (1:100; GB111401, Servicebio, Wuhan, China). Livers embedded in an optimum cutting temperature compound were used for oil red 0 staining for assessment of hepatic steatosis. The procedure was performed as previously detailed and observed under an optical microscope (magnification 400x). All tissues were evaluated by two experienced and 'blinded' pathologists and the histological scoring system for Nonalcoholic steatohepatitis (NASH) was 
conducted according to the NAS score system: 0 = basically no inflammation, $1 \leq 400$-fold field of view, 2 $=400-200$-fold field of view, $3 \geqslant 200-100$-fold field of view, $4=$ up to the 40 -fold field of view. Color thresholding and measurement of area fraction with ImageJ was also utilized (National Institutes of Health, Bethesda, MD).

\subsection{Determination of triglyceride (TG) and cholesterol (CHO) in liver}

$100 \mathrm{mg}$ of liver samples were weighed and then $0.9 \mathrm{~mL}$ of absolute ethanol was added in to the centrifuge tube. Afterwards, the liver samples were homogenized with an electric homogenizer upon ice water and centrifuged at $4^{\circ} \mathrm{C} 2500 \mathrm{r} / \mathrm{min}$ for $10 \mathrm{~min} .2 .5 \mu$ of supernatant for TG determination was acquired. The TG content was determined by using a commercial kit (Nanjing Jiancheng Bioengineering Institute) and the cholesterol was measured by a cholesterol assay kit (Nanjing Jiancheng Bioengineering Institute). The final concentrations of triglycerides and cholesterol were corrected for protein content.

\subsection{DNA Amplification and High-Throughput Sequencing}

Fecal samples were collected immediately upon defecation from each rat at baseline (1st week and 16th week) and stored at $-80^{\circ} \mathrm{C}$.

E.Z.N.A.® soil DNA Kit (Omega Bio-Tek, Norcross, GA, U.S.) was then employed to extract microbial DNA from ileum according to the manufacturer's protocols.

The NanoDrop 2000 UV-vis spectrophotometer (Thermo Scientific, Wilmington, USA) was also utilized to determine the final DNA concentration and purification. The amplification of the v3-v4 region of the bacteria 16S rRNA gene was conducted with universal primer pairs (338F 5'-

ACTCCTACGGGAGGCAGCAG-3' and 806R 5'-ACTCCTACGGGAGGCAGCAG-3'). PCR was performed in a 20uL amplification system. The PCR program as follows: denaturation at $95^{\circ} \mathrm{C}$ for $3 \mathrm{~min}, 27$ cycles at $95^{\circ} \mathrm{C}$ for $30 \mathrm{~s}$, annealing at $55^{\circ} \mathrm{C}$ for $30 \mathrm{~s}, 4$ elongations at $72^{\circ} \mathrm{C}$ for $5 \mathrm{~s}$, and a final extension at $72^{\circ} \mathrm{C}$ for 10 min. Purified amplicons were pooled in equimolar and paired-end sequenced $(2 \times 300)$ on an Illumina MiSeq platform (Illumina, San Diego, USA) in accordance with the standard protocols by Majorbio BioPharm Technology Co. Ltd. (Shanghai, China) ${ }^{52}$. Sequencing data, including the filtration of adapter sequences, low-quality reads, and ambiguous base were then processed. These sequences were clustered into operational taxonomic units (OTUs) with a $97 \%$ sequence identity using MOTHUR (furthest neighbor method) and chopSeq (Majorbio).

\subsection{Determination of LPS in the plasma}

Blood samples were collected from the portal vein and then centrifuged at $4^{\circ} \mathrm{C} 2500 \mathrm{r} / \mathrm{min}$ for $5 \mathrm{~min}$. Plasma was transferred into LPS-free cups and stored at $-20^{\circ} \mathrm{C}$ for analysis. The content of LPS in plasma was detected by an endpoint chromogenic method utilizing a test kit.

\subsection{Western Blotting}


The liver tissue was lysed in RIPA with 1\% PMSF and the protein was then fractionated by electrophoresis on $10 \%$ SDS-PAGE. Then, the protein was transferred to the PVDF membrane. Western blotting was used to determine the expression of TLR4 (Proteintech Group, Chicago, IL, USA), MYD88 (Proteintech Group, Chicago, IL, USA) and AP-1(Proteintech Group, Chicago, IL, USA), with GAPDH Antibody (Proteintech Group, Chicago, IL, USA) as the internal reference. The ratio of phosphorylated NF-kB p65 protein (Cell Signaling Technology, Danvers, MA, USA) to total NF-KB p65 protein (Proteintech Group, Chicago, IL, USA) content indicated its phosphorylation level. The antibody-antigen complexes were visualized by employing the ECL (Electrochemiluminescence) kit.

\subsection{Determination of inflammatory cytokines (IL-1 $\beta$, IL-6, TNF-a) in liver and plasma}

$100 \mathrm{mg}$ of liver samples were weighed and then $0.9 \mathrm{~mL}$ of physiological saline was added into the centrifuge tube. Afterwards, the liver tissue was homogenized with an electric homogenizer upon ice water and centrifuged at $4^{\circ} \mathrm{C} 2500 \mathrm{r} / \mathrm{min}$ for $20 \mathrm{~min} .10 \mu$ of supernatant was taken for corresponding inflammatory cytokines (IL-1 $\beta, \mathrm{IL}-6, \mathrm{TNF}-\alpha)$ and determined each time. The inflammatory cytokines (IL-1 $\beta$, IL-6, TNF- $\alpha$ ) content were determined by specific ELISA kits according to the manufacturer's instructions (Beijing Winter Song Boye Biotechnology Co. Ltd.).

IL-1 $\beta$, IL-6, and TNF-a in plasma were measured by enzyme-linked immunosorbent assay (ELISA), according to the recommended procedures on the kits (IL-1 $\beta$, lot No.

A301B00631, MULTI Sciences; IL-6, lot No. A30600115, MULTI Sciences; TNF-a, lot No. 222131-001, invitrogen., USA).

\subsection{Statistical analysis}

Data were analyzed by using SPSS 21.0 software and data that conformed to the normal distribution were expressed as the mean \pm SD. T-test was used for comparison between two groups, and one-way analysis of variance was utilized for comparison among multiple groups. P-value $<0.05$ was considered to be significantly different.

\section{Declarations}

\section{Acknowledgments}

This work was supported by the National Natural Science Foundation of China (No.81673660, 81873242)

\section{Conflict of Interests}

The authors declare no conflict of interests.

\section{References}


1. Chalasani, N. et al. The Diagnosis and Management of Non-alcoholic Fatty Liver Disease: Practice Guideline by the American Gastroenterological Association, American Association for the Study of Liver Diseases, and American College of Gastroenterology. Gastroenterology. 142, 1592-1609 https://doi.org/10.1053/j.gastro.2012.04.001 (2012).

2. Brown, G. T. \& Kleiner, D. E. Histopathology of nonalcoholic fatty liver disease and nonalcoholic steatohepatitis. Metabolism. 65, 1080-1086 https://doi.org/10.1016/j.metabol.2015.11.008 (2016).

3. Younossi, Z. M. et al. Global Epidemiology of Nonalcoholic Fatty Liver Disease-Meta-Analytic Assessment of Prevalence, Incidence, and Outcomes. Hepatology. 64, 73-84 https://doi.org/10.1002/hep.28431 (2016).

4. Sayiner, M., Koenig, A., Henry, L. \& Younossi, Z. M. Epidemiology of Nonalcoholic Fatty Liver Disease and Nonalcoholic Steatohepatitis in the United States and the Rest of the World. Clin Liver Dis. 20, 205-205 https://doi.org/10.1016/j.cld.2015.10.001 (2016).

5. Than, N. N. \& Newsome, P. N. A concise review of non-alcoholic fatty liver disease. Atherosclerosis. 239, 192-202 https://doi.org/10.1016/j.atherosclerosis.2015.01.001 (2015).

6. Banini, B. A. \& Sanyal, A. J. Current and future pharmacologic treatment of nonalcoholic steatohepatitis. Curr Opin Gastroen. 33, 134-141 https://doi.org/10.1097/Mog.0000000000000356 (2017).

7. Dulai, P. S. et al. Increased Risk of Mortality by Fibrosis Stage in Nonalcoholic Fatty Liver Disease: Systematic Review and Meta-Analysis. Hepatology. 65, 1557-1565 https://doi.org/10.1002/hep.29085 (2017).

8. Rinella, M. E. Nonalcoholic Fatty Liver Disease A Systematic Review. Jama-J Am Med Assoc. 313, 2263-2273 https://doi.org/10.1001/jama.2015.5370 (2015).

9. Wu, D., Chen, X., Hu, S. \& Bai, X. H. Study on major antitumor components in Yinchenhao decoction in vitro and in vivo based on hollow fiber cell fishing coupled with high performance liquid chromatography. J Chromatogr B. 1060, 118-125 https://doi.org/10.1016/j.jchromb.2017.06.003 (2017).

10. Jin, S. et al. Chlorogenic acid improves late diabetes through adiponectin receptor signaling pathways in db/db mice. Plos One. 10, e0120842 https://doi.org/10.1371/journal.pone.0120842 (2015).

11. Bhandarkar, N. S., Brown, L. \& Panchal, S. K. Chlorogenic acid attenuates high-carbohydrate, high-fat diet-induced cardiovascular, liver, and metabolic changes in rats. Nutr Res. 62, 78-88 (2019).

12. Huang, S. et al. Chlorogenic acid effectively treats cancers through induction of cancer cell differentiation. Theranostics. 9, 6745-6763 https://doi.org/10.7150/thno.34674 (2019).

13. He, T. et al. Geniposide attenuates cadmiuminduced oxidative stress injury via Nrf2 signaling in osteoblasts. Mol Med Rep. 20, 1499-1508 https://doi.org/10.3892/mmr.2019.10396 (2019).

14. Jiang, Y. Q. et al. Geniposide Prevents Hypoxia/Reoxygenation-Induced Apoptosis in H9c2 Cells: Improvement of Mitochondrial Dysfunction and Activation of GLP-1R and the PI3K/AKT Signaling Pathway. Cell Physiol Biochem. 39, 407-421 https://doi.org/10.1159/000445634 (2016). 
15. Shi, H. T. et al. Chlorogenic acid protects against liver fibrosis in vivo and in vitro through inhibition of oxidative stress. Clin Nutr. 35, 1366-1373 https://doi.org/10.1016/j.clnu.2016.03.002 (2016).

16. Feng, Y. et al. Chlorogenic acid protects $d$-galactose-induced liver and kidney injury via antioxidation and anti-inflammation effects in mice. Pharm Biol. 54, 1027-1034 https://doi.org/10.3109/13880209.2015.1093510 (2016).

17. Yao, X. Y. et al. Leukadherin-1-Mediated Activation of CD11b Inhibits LPS-Induced Pro-inflammatory Response in Macrophages and Protects Mice Against Endotoxic Shock by Blocking LPS-TLR4 Interaction. Front Immuno/10, doi:ARTN 215 3389/fimmu.2019.00215 (2019).

18. Tian, X. et al. Exploration of the hepatoprotective chemical base of an orally administered herbal formulation (YCHT) in normal and CCl4-intoxicated liver injury rats. Part 2: Hepatic disposition in vivo and hepatoprotective activity in vitro. Journal of ethnopharmacology. 236, 161-172 https://doi.org/10.1016/j.jep.2019.02.022 (2019).

19. Xu, B. et al. Geniposide ameliorates TNBS-induced experimental colitis in rats via reducing inflammatory cytokine release and restoring impaired intestinal barrier function. Acta Pharmacol Sin. 38, 688-698 https://doi.org/10.1038/aps.2016.168 (2017).

20. Liang, H. et al. Optimization of dosage ratio of chlorogenic acid and gardenia glycosides in the treatment of rats with fatty liver disease induced by high-fat feed. J Tradit Chin Med. 36, 683-688 https://doi.org/10.1016/s0254-6272(16)30090-5 (2016).

21. Liang, H. et al. Inhibitory Effect of Gardenoside on Free Fatty Acid-Induced Steatosis in HepG2 Hepatocytes. Int J Mol Sci. 16, 27749-27756 https://doi.org/10.3390/ijms161126058 (2015).

22. Chen, S. D. et al. Study on effects of zhi zi (fructus gardeniae) on non-alcoholic fatty liver disease in the rat. J Tradit Chin Med. 32, 82-86 https://doi.org/10.1016/s0254-6272(12)60037-5 (2012).

23. Buzzetti, E., Pinzani, M. \& Tsochatzis, E. A. The multiple-hit pathogenesis of non-alcoholic fatty liver disease (NAFLD). Metabolism. 65, 1038-1048 https://doi.org/10.1016/j.metabol.2015.12.012 (2016).

24. Ghetti, F. D. et al. Influence of gut microbiota on the development and progression of nonalcoholic steatohepatitis. Eur J Nutr. 57, 861-876 https://doi.org/10.1007/s00394-017-1524-x (2018).

25. Wiest, R., Albillos, A., Trauner, M., Bajaj, J. S. \& Jalan, R. Targeting the gut-liver axis in liver disease. J Hepatol. 67, 1084-1103 https://doi.org/10.1016/j.jhep.2017.05.007 (2017).

26. Cani, P. D. et al. Changes in gut microbiota control metabolic endotoxemia-induced inflammation in high-fat diet-induced obesity and diabetes in mice. Diabetes. 57, 1470-1481 https://doi.org/10.2337/db07-1403 (2008).

27. Han, M. S. et al. JNK expression by macrophages promotes obesity-induced insulin resistance and inflammation. Science. 339, 218-222 https://doi.org/10.1126/science.1227568 (2013).

28. Adolph, T. E., Grander, C., Moschen, A. R. \& Tilg, H. Liver-Microbiome Axis in Health and Disease. Trends Immunol. 39, 712-723 https://doi.org/10.1016/j.it.2018.05.002 (2018).

29. Clemente, J. C., Manasson, J. \& Scher, J. U. The role of the gut microbiome in systemic inflammatory disease. BMJ. 360, j5145 https://doi.org/10.1136/bmj.j5145 (2018). 
30. Iacono, A., Raso, G. M., Canani, R. B., Calignano, A. \& Meli, R. Probiotics as an emerging therapeutic strategy to treat NAFLD: focus on molecular and biochemical mechanisms. J Nutr Biochem. 22, 699-711 https://doi.org/10.1016/j.jnutbio.2010.10.002 (2011).

31. Xue, L. et al. Probiotics may delay the progression of nonalcoholic fatty liver disease by restoring the gut microbiota structure and improving intestinal endotoxemia. Sci Rep-Uk 7, doi:Artn 45176 1038/Srep45176 (2017).

32. Tarantino, G. \& Finelli, C. Systematic review on intervention with prebiotics/probiotics in patients with obesity-related nonalcoholic fatty liver disease. Future Microbiol. 10, 889-902 https://doi.org/10.2217/Fmb.15.13 (2015).

33. Liu, J. et al. Geniposide reduces cholesterol accumulation and increases its excretion by regulating the FXR-mediated liver-gut crosstalk of bile acids. Pharmacol Res. 152, 104631 https://doi.org/10.1016/j.phrs.2020.104631 (2020).

34. Fan, X. et al. Therapeutic potential of genipin in various acute liver injury, fulminant hepatitis, NAFLD and other non-cancer liver diseases: More friend than foe. Pharmacol Res. 159, 104945 https://doi.org/10.1016/j.phrs.2020.104945 (2020).

35. Shen, B. et al. Geniposide alleviates non-alcohol fatty liver disease via regulating Nrf2/AMPK/mTOR signalling pathways. J Cell Mol Med. 24, 5097-5108 https://doi.org/10.1111/jcmm.15139 (2020).

36. Li, J. Y. et al. Therapeutic mechanism of Yin-Chen-Hao decoction in hepatic diseases. World J Gastroenterol. 23, 1125-1138 https://doi.org/10.3748/wjg.v23.i7.1125 (2017).

37. Reagan-Shaw, S., Nihal, M. \& Ahmad, N. Dose translation from animal to human studies revisited. Faseb J. 22, 659-661 https://doi.org/10.1096/fj.07-9574LSF (2008).

38. Shyangdan, D. et al. Insulin sensitisers in the treatment of non-alcoholic fatty liver disease: a systematic review. Health Technol Asses. 15, 1-1 https://doi.org/10.3310/hta15380 (2011).

39. Yu, H. et al. Genome Sequence of the Bacterium Bifidobacterium longum Strain CMCC P0001, a Probiotic Strain Used for Treating Gastrointestinal Disease. Genome Announc. 1, https://doi.org/10.1128/genomeA.00716-13 (2013).

40. Cui, H. H. et al. Effects of probiotic on intestinal mucosa of patients with ulcerative colitis. World J Gastroentero. 10, 1521-1525 (2004).

41. du Preez, R. et al. Carrageenans from the Red Seaweed Sarconema filiforme Attenuate Symptoms of Diet-Induced Metabolic Syndrome in Rats. Mar Drugs. 18, https://doi.org/10.3390/md18020097 (2020).

42. Zhang, F. et al. Bene fi cial effects of probiotic cholesterol-lowering strain of Enterococcus faecium WEFA23 from infants on diet-induced metabolic syndrome in rats. J Dairy Sci. 100, 1618-1628 https://doi.org/10.3168/jds.2016-11870 (2017).

43. Yu, J. et al. Gancao-Gansui combination impacts gut microbiota diversity and related metabolic functions. J Ethnopharmacol. 214, 71-82 https://doi.org/10.1016/j.jep.2017.11.031 (2018).

44. Turnbaugh, P. J. et al. An obesity-associated gut microbiome with increased capacity for energy harvest. Nature. 444, 1027-1031 https://doi.org/10.1038/nature05414 (2006). 
45. Abdallah Ismail, N. et al. Frequency of Firmicutes and Bacteroidetes in gut microbiota in obese and normal weight Egyptian children and adults. Arch Med Sci. 7, 501-507 https://doi.org/10.5114/aoms.2011.23418 (2011).

46. Gerritsen, J. et al. Genomic and functional analysis of Romboutsia ilealis CRIB(T) reveals adaptation to the small intestine. PeerJ. 5, e3698 https://doi.org/10.7717/peerj.3698 (2017).

47. Manzhalii, E., Virchenko, O., Falalyeyeva, T., Beregova, T. \& Stremmel, W. Treatment efficacy of a probiotic preparation for non-alcoholic steatohepatitis: A pilot trial. J Dig Dis. 18, 698-703 https://doi.org/10.1111/1751-2980.12561 (2017).

48. Rivera, C. A. et al. Toll-like receptor-4 signaling and Kupffer cells play pivotal roles in the pathogenesis of non-alcoholic steatohepatitis. $J$ Hepatol. 47, 571-579 https://doi.org/10.1016/j.jhep.2007.04.019 (2007).

49. Zhan, Y. T. \& An, W. Roles of liver innate immune cells in nonalcoholic fatty liver disease. World J Gastroentero. 16, 4652-4660 https://doi.org/10.3748/wjg.v16.i37.4652 (2010).

50. Miura, K. et al. Toll-Like Receptor 9 Promotes Steatohepatitis by Induction of Interleukin-1 beta in Mice. Gastroenterology. 139, 323-453 https://doi.org/10.1053/j.gastro.2010.03.052 (2010).

51. Kucera, O. \& Cervinkova, Z. Experimental models of non-alcoholic fatty liver disease in rats. World $J$ Gastroentero. 20, 8364-8376 https://doi.org/10.3748/wjg.v20.i26.8364 (2014).

52. Schloss, P. D. et al. Introducing mothur: open-source, platform-independent, community-supported software for describing and comparing microbial communities. App/ Environ Microbiol. 75, 75377541 https://doi.org/10.1128/AEM.01541-09 (2009).

\section{Figures}



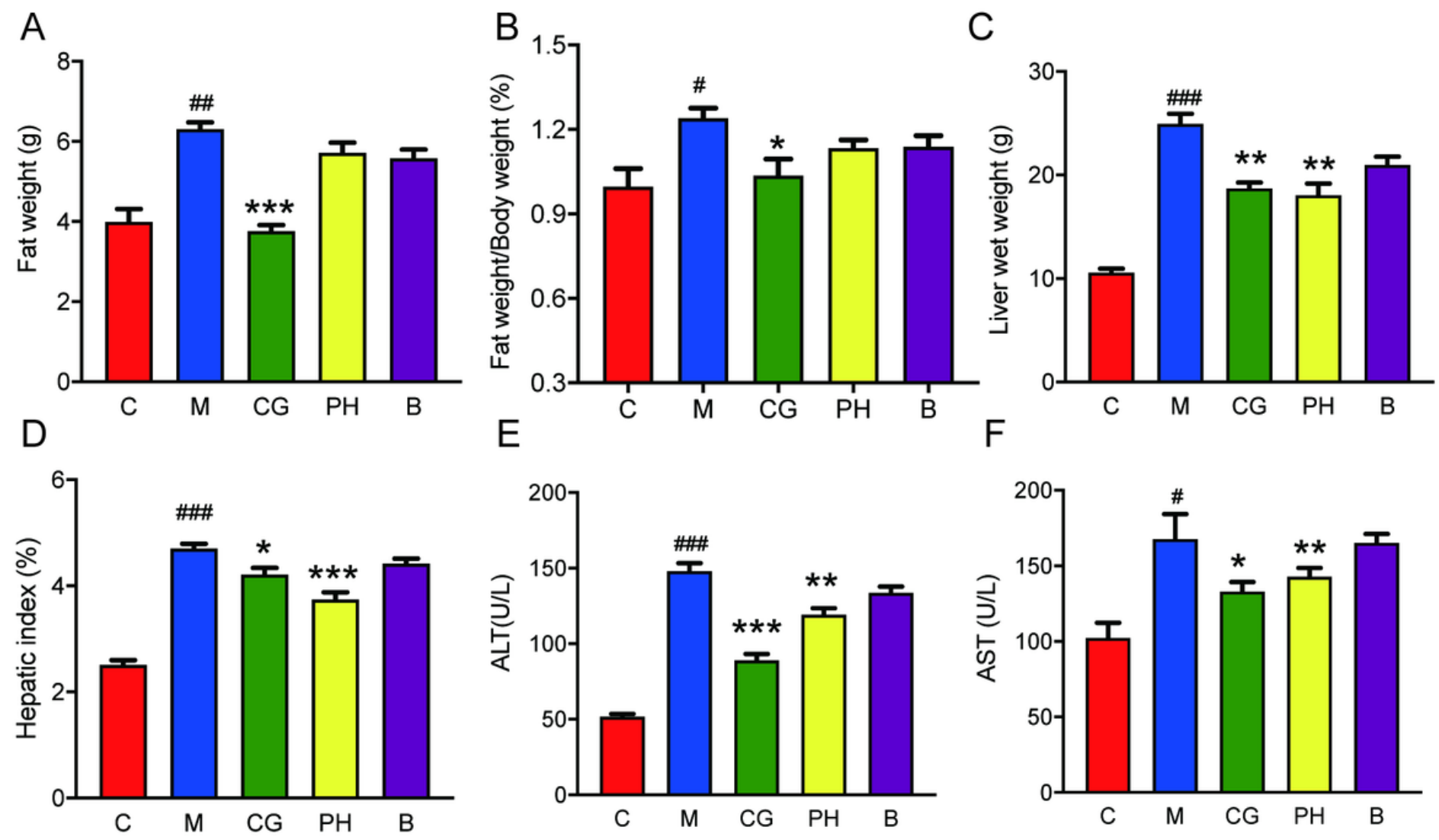

Figure 1

Articulates the effect of $\mathrm{CG}$ on body weight, obesity and serum transaminases. A. Fat weight of rats in epididymal tissue at the 16th week. B. Fat weight/body weight of Rats at the 16th week. C. The liver wet weight of rats at week 16. D. Hepatic index (hepatic index = liver weight/body weight $\times 100$ ) of rats. The effect of CG on the liver injury was determined by detecting the serum levels of ALT(E) and AST(F).

Statistical analyses were conducted with one-way ANOVA. \#P $<0.05$, \#\#P<0.01, \#\#\#P<0.001 versus C; $\star P<0.05,{ }^{*} \mathrm{P}<0.01, * \star \star \mathrm{P}<0.001$ versus $\mathrm{M}$. 
A

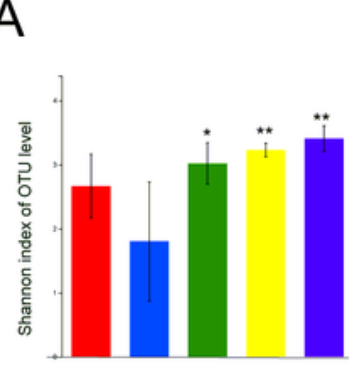

C $M \quad C G \quad P H \quad B$

E

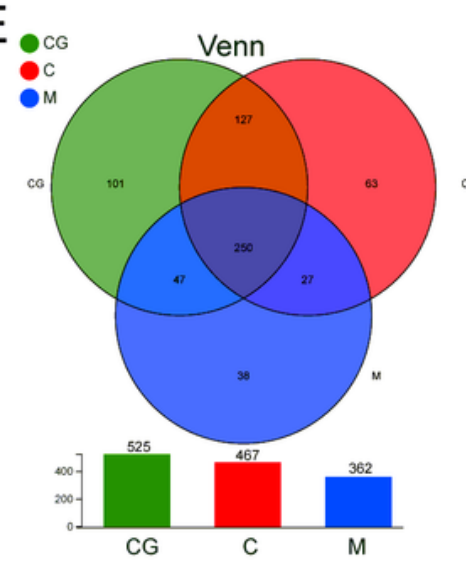

G

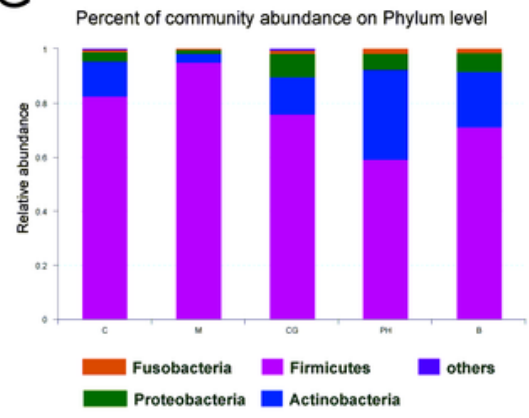

J

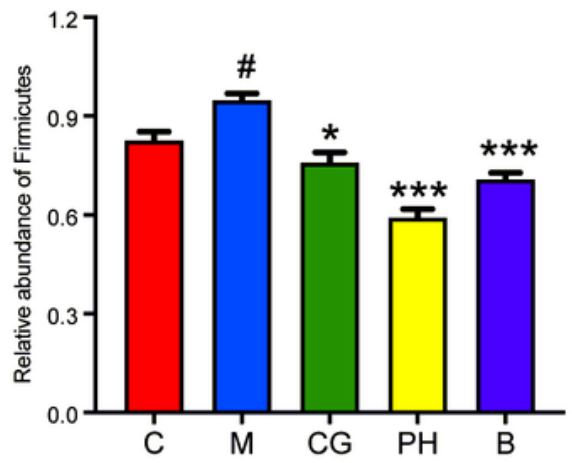

F
B weighted_unifrac PCOA
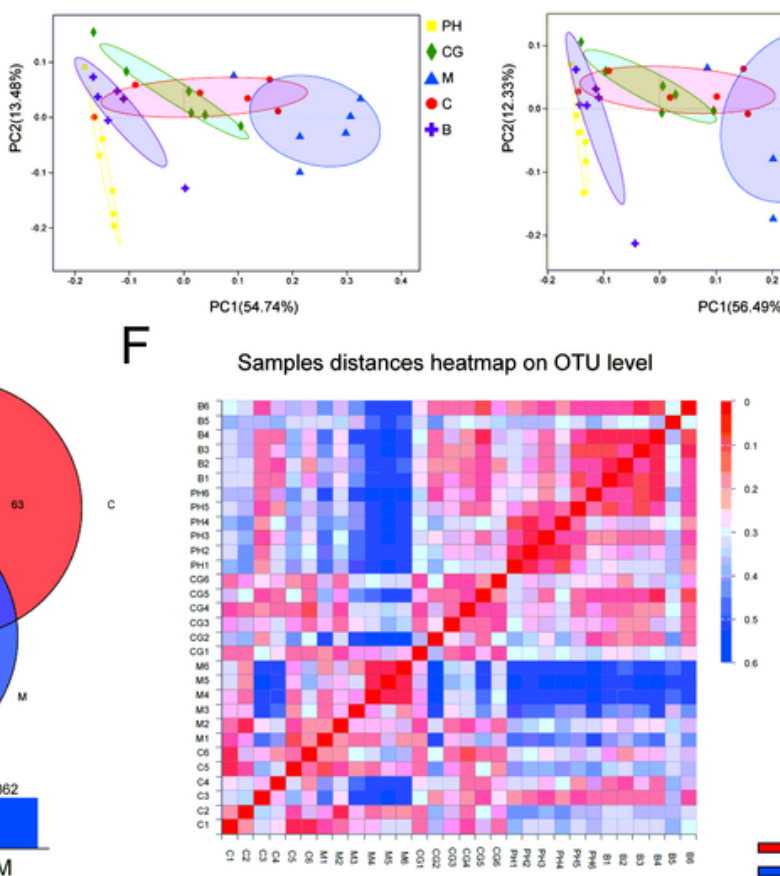

$\mathrm{H}$

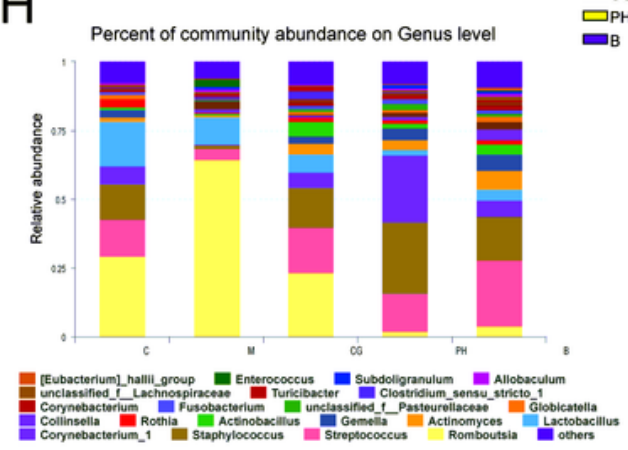

$\mathrm{K}$

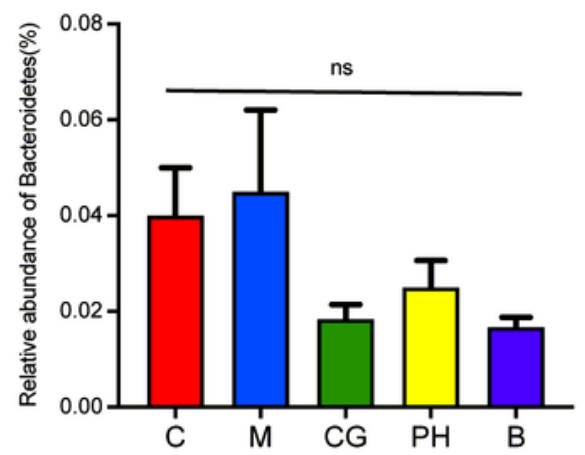

D

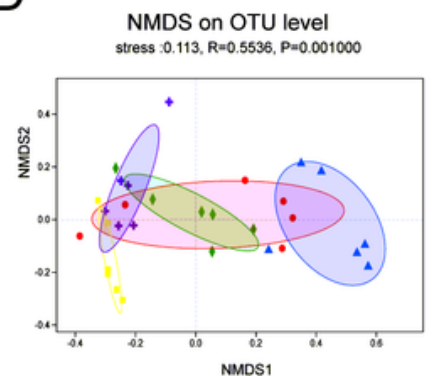

I
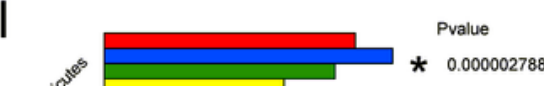

* $8.919 \mathrm{e}-7$

0.1024

* 0.0482

0.2827

$\begin{array}{lccccccccc} & 1 & 1 & 1 & 1 & 1 & 1 & 1 & 1 & 1 \\ 0 & 10 & 20 & 30 & 40 & 50 & 60 & 70 & 80 & 90\end{array}$

Mean proportions(\%)
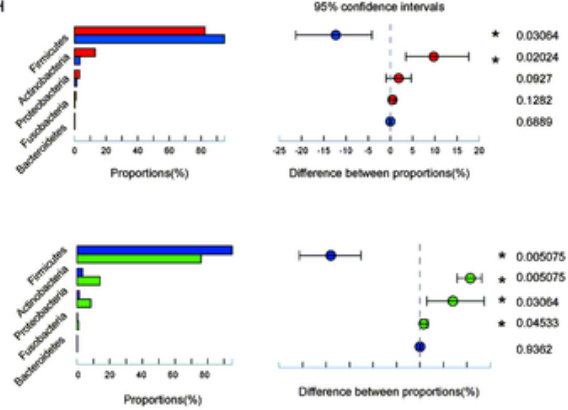

L

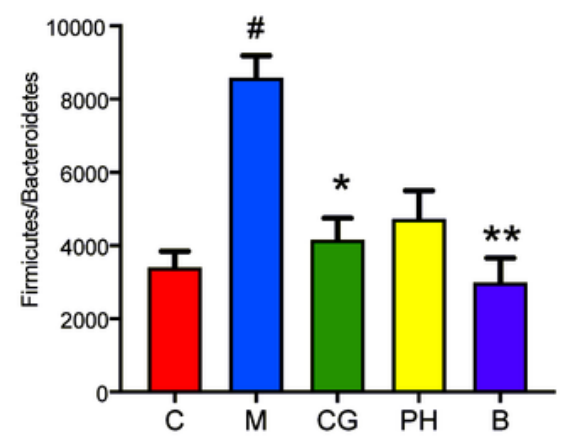

Figure 2

exhibits the influences of CG on intestinal microbiota. (A) OTU levels by Shannon diversity index in each group. (B, C) Scatter plots of the unweighted-UniFrac principal coordinates analysis (PCOA) score showing the similarity of the 30 bacterial communities based on the UniFrac distance and Scatter plot of the weighted-PCOA score. (D) Nonmetric multidimensional scaling (NMDS) showing the difference in bacterial communities according to the Bray-Curtis distance. (E) Venn diagram representing the number 
of OTUs among the C, M and CG groups. (F) Heatmap of the hierarchical cluster analysis. $(G, H)$ The bacterial composition of the different communities at the genus level $(G)$ and phylum level $(H)$. (I) Details of the differences in the genus level of the microbiota in each group. The relative abundance of firmicutes $(\mathrm{J})$ and Bacteroidetes $(\mathrm{K})$ in each group. $(\mathrm{L})$ The ratio of Firmicutes/Bacteroidetes. Statistical analyses

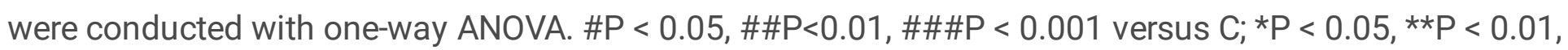
$\star \star * \mathrm{P}<0.001$ versus $\mathrm{M}$.

A

$\mathrm{C}$

M

CG

$\mathrm{PH}$

B
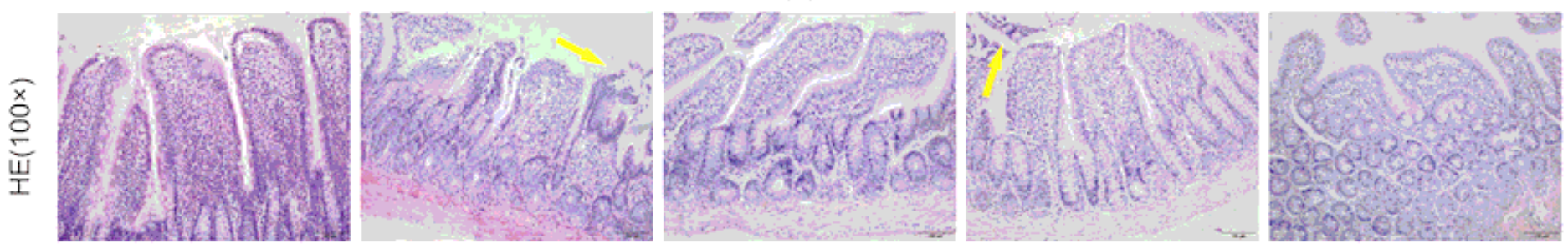

$\mathrm{B}$
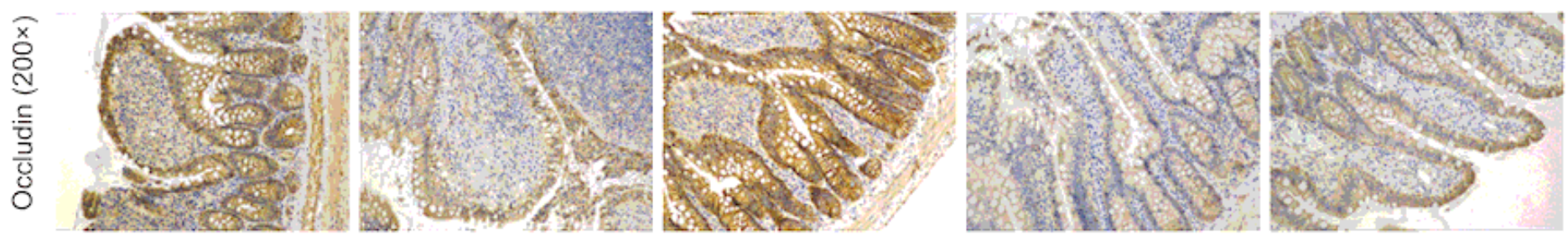

C
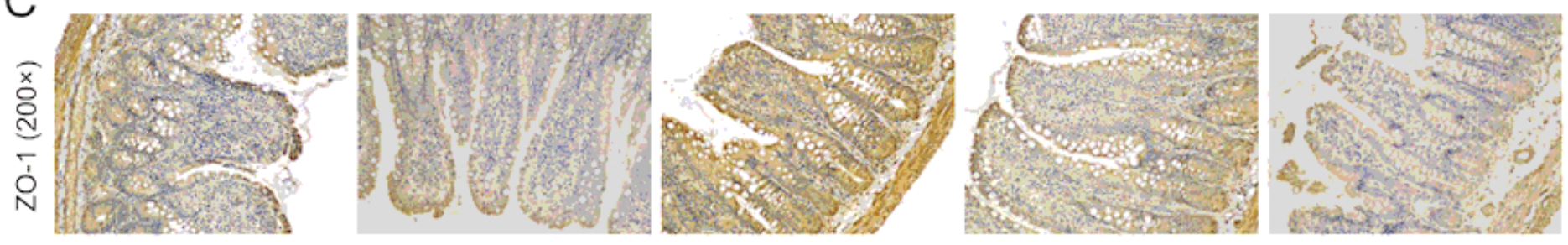

$\mathrm{D}$

$E$
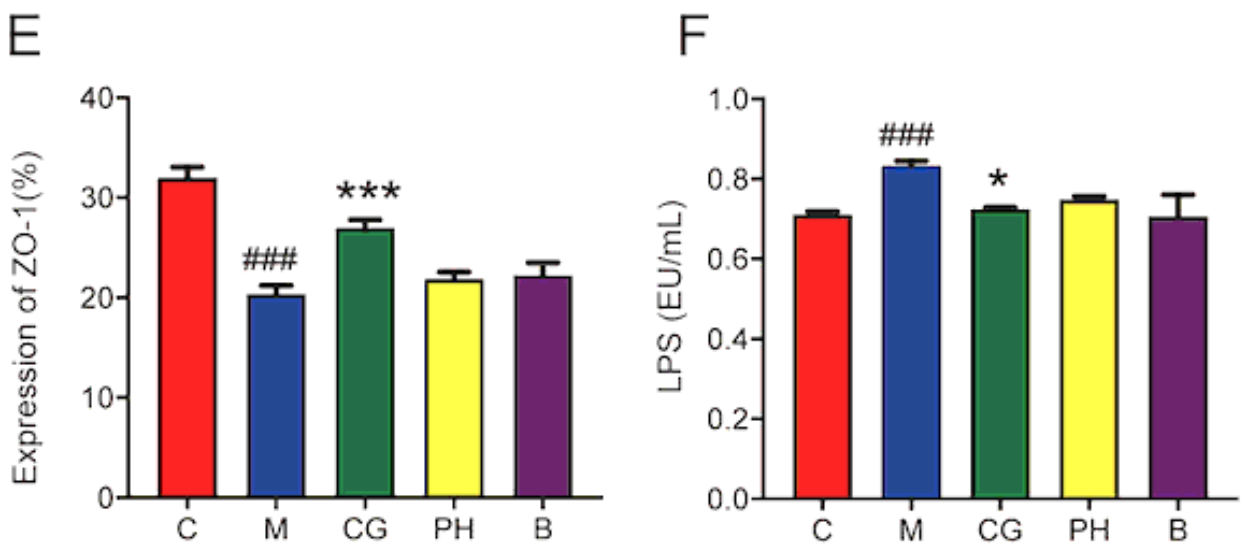

\section{Figure 3}

Beneficial effects of CG on the terminal ileum and the level of serum LPS. (A) Formalin-fixed and paraffinembedded terminal ileum of the C, M, CG, PH and B groups were stained with hematoxylin \& eosin (HE) and observed under an optical microscope $(\mathrm{HE}, \times 400)$, the yellow arrow marks the shed intestinal epithelial cells. (B) Representative immunostaining of Occludin (B) and zonula occluden-1 (ZO-1) (C), 
respectively. (D, E) The expression of Occludin (D) and ZO-1(E) of terminal ileum analyzed with ImageJ $(F)$ the LPS content in plasma. Statistical analyses were conducted with one-way ANOVA. \#P $<0.05$, $\# \# P<0.01, \# \# \# P<0.001$ versus $C ; * P<0.05,{ }^{*} \mathrm{P}<0.01$, $* \star * P<0.001$ versus $\mathrm{M}$.

A
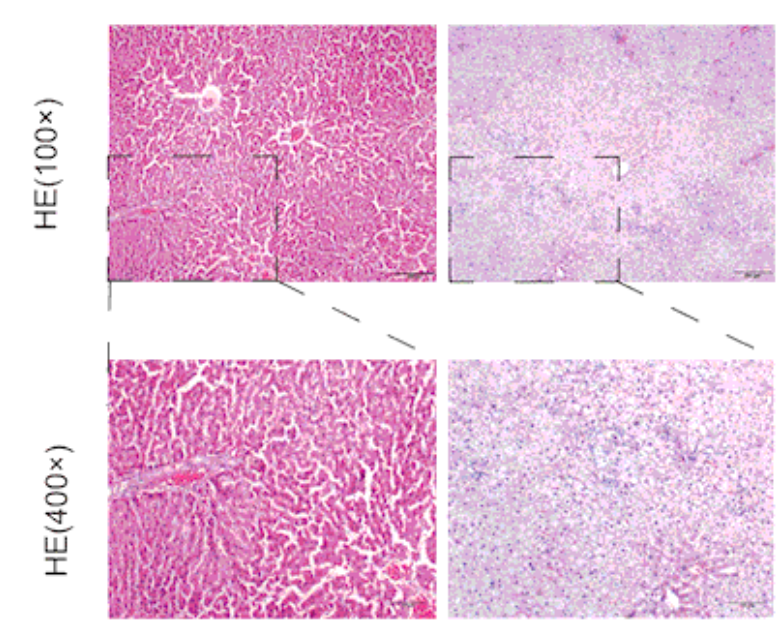

CG

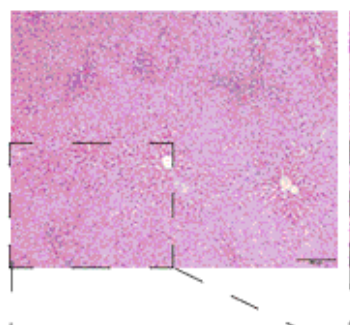

$\mathrm{PH}$

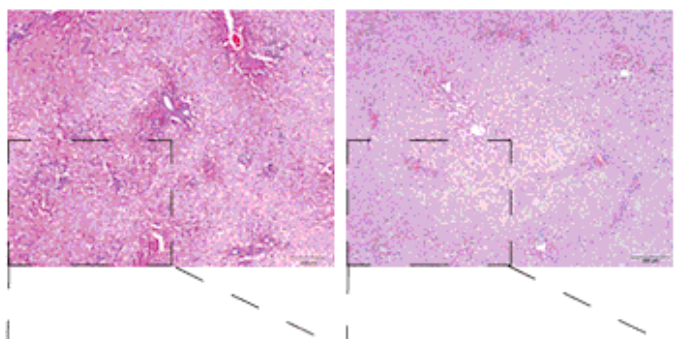

B
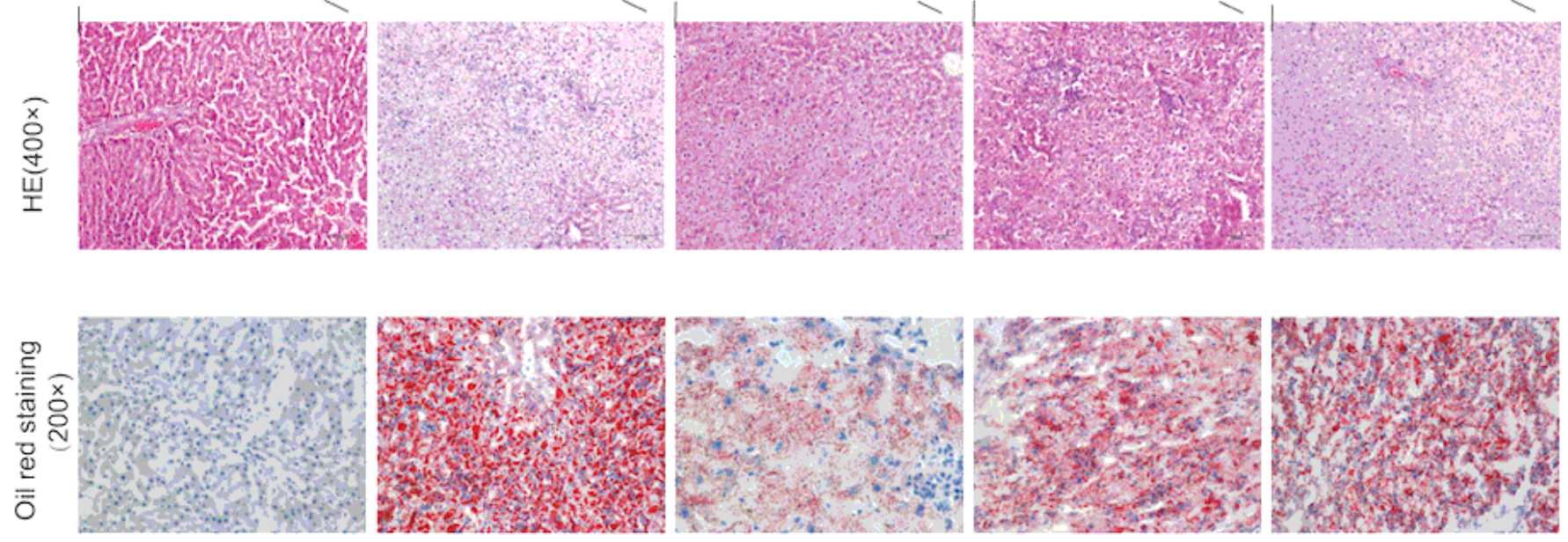

C

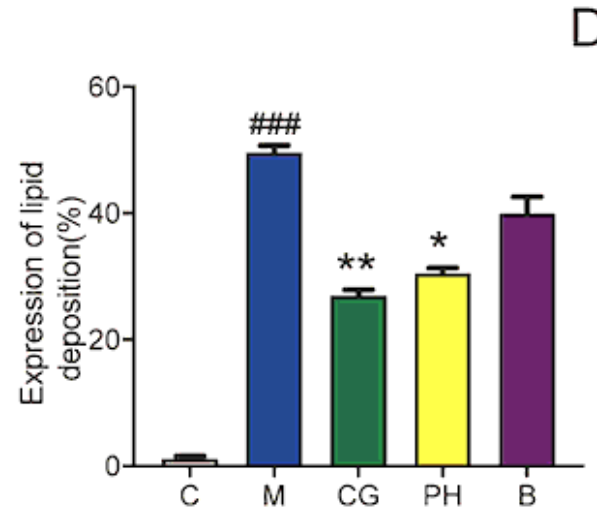

$\mathrm{D}$

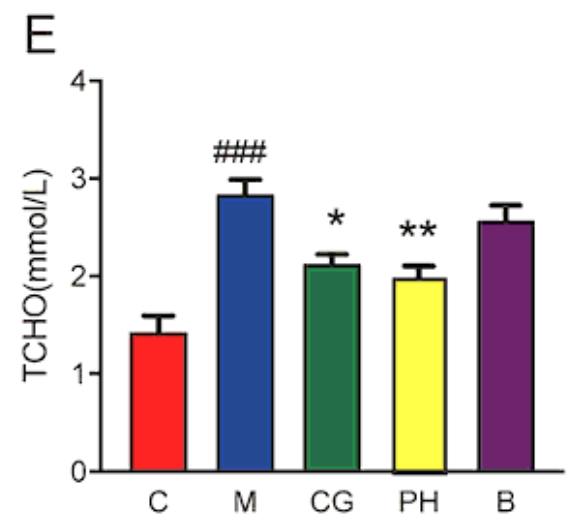

Figure 4

CG improves the pathology of the liver tissue. (A) Formalin-fixed and paraffin-embedded liver of the $C$ group, M group, CG group, PH group and B group were stained with hematoxylin \& eosin (HE) and observed under an optical microscope $(\mathrm{HE}, \times 100$, and $\times 400)$. Black arrows indicate steatosis of hepatocytes, red arrows indicate dotted necrosis areas. (B) Representative oil red $O$ staining of the liver. (C) The expression of lipid deposition of liver analyzed with ImageJ. (D, E) The level of TG and cholesterol in the liver. Statistical analyses were conducted with one-way ANOVA. \#P $<0.05, \# \# P<0.01, \# \# \# P<0.001$ versus $C$; $* P<0.05,{ }^{\star} \mathrm{P}<0.01,{ }^{\star} * \star \mathrm{P}<0.001$ versus $\mathrm{M}$. 
A

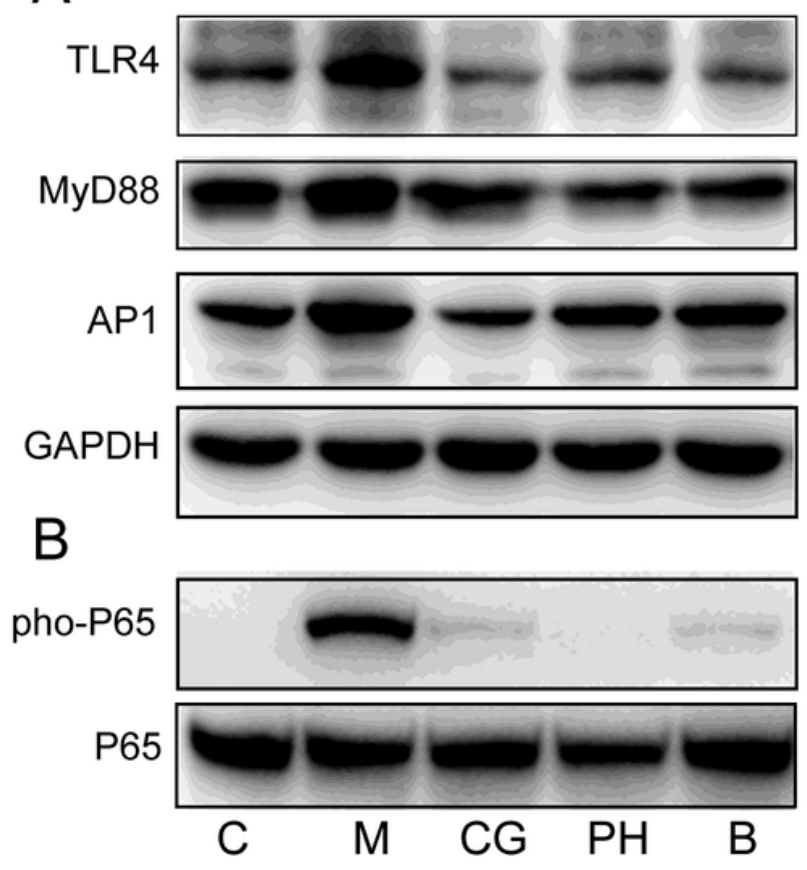

C
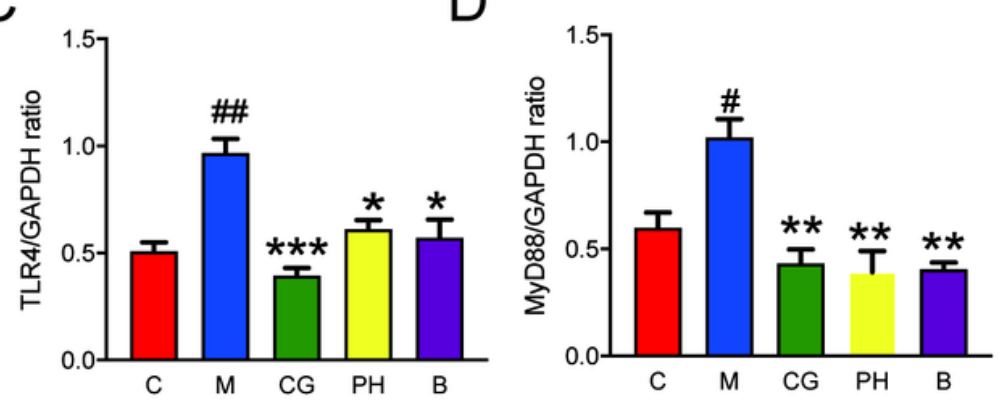

$E$

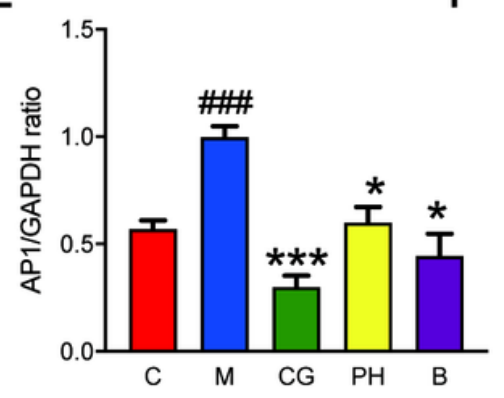

$\mathrm{F}$

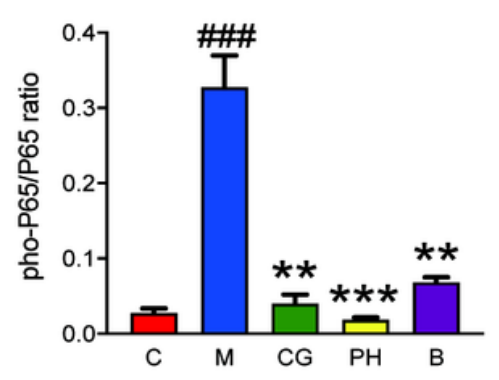

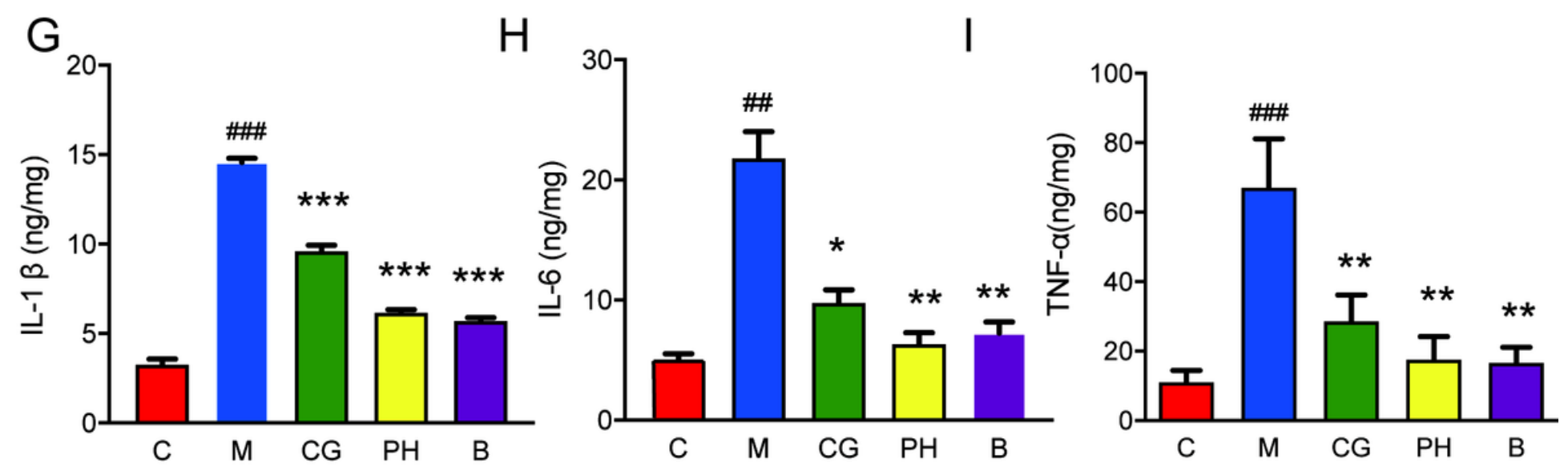

Figure 5

The effect of CG on LPS-TLR4 signaling pathway activation in HFD-fed rats. (A) Representative western blots of TLR4, AP-1 and MyD88 protein expression in the liver are shown for each group. GAPDH was used as a loading control. (C-E) Bar graphs show densitometry analysis of specific bands expressed as percentages relative to control. (B)A representative western blot of phosphorylated NF-KB p65 in the liver of each group is shown. Total NF-KB p65 was used as a loading control, indicating its phosphorylation level. (F) Bar graphs show densitometry analysis of specific bands expressed as percentages relative to the control. (G-I) CG decreased the content of inflammatory cytokines (IL-1 $\beta$, IL-6, TNF- $\alpha$ ) in the liver. The content of IL-1 $\beta$, IL-6, and TNF-a in each group. The LPS content in plasma. Statistical analyses were

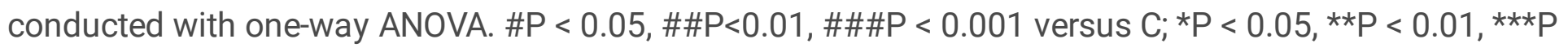
$<0.001$ versus $M$. 


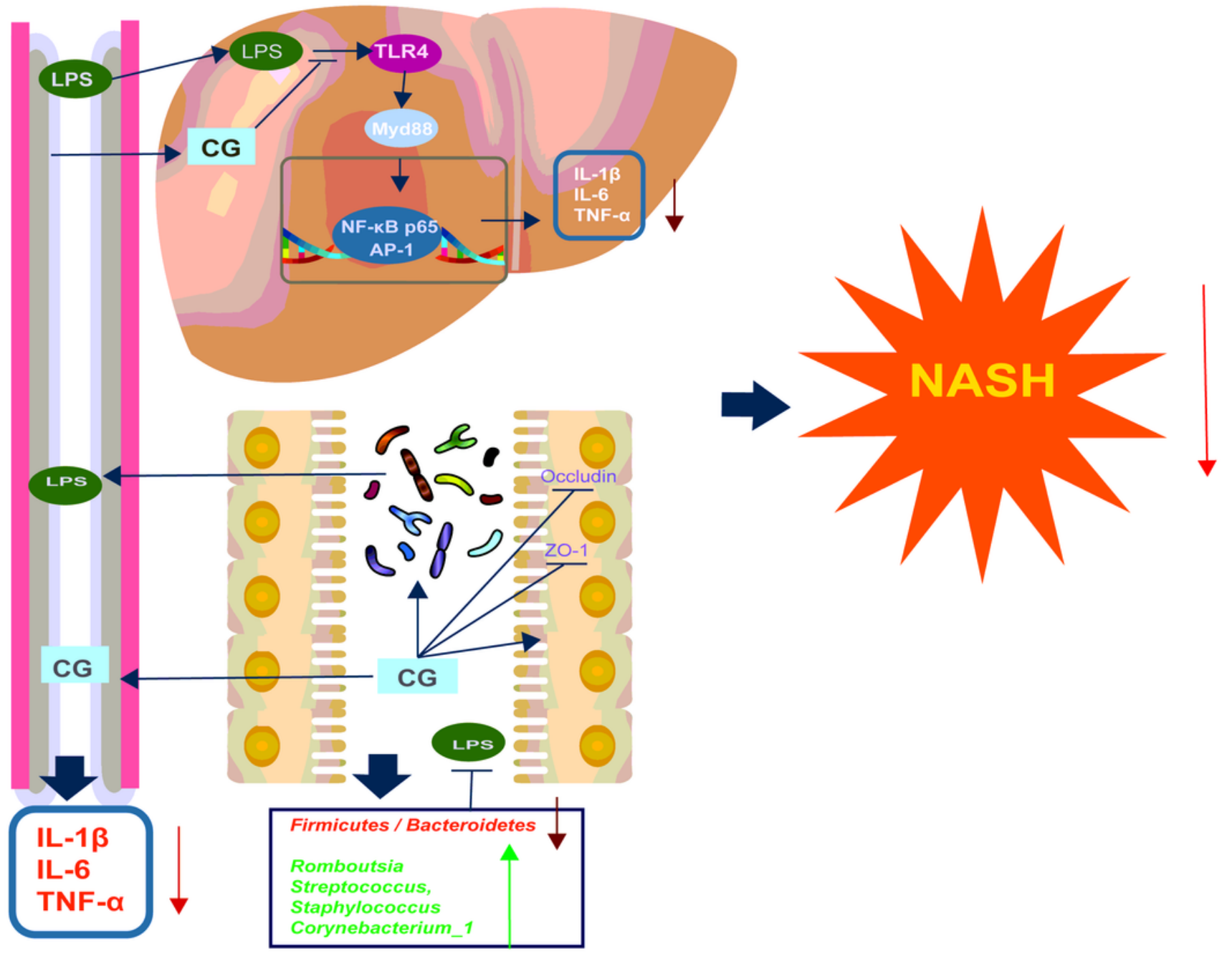

Figure 6

The beneficial effects of CG on the gut microbiota and steatohepatitis. Administration of CG improves microbiota dysbiosis, increases tight junction proteins in the gut, and decreases the production of endotoxin. CG also attenuates NASH through inhibiting activation of TLR4-LPS signaling and the production of proinflammatory cytokines. 